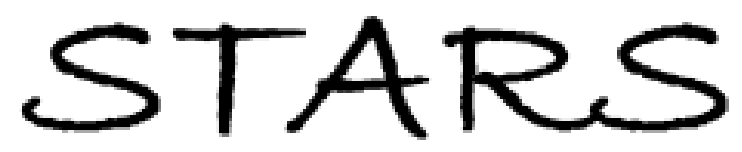

University of Central Florida

STARS

$1-1-2013$

\title{
Monolithic quantum-well-tunable laser based on optical beam steering and area-selective intermixing
}

\author{
Abdullah Zakariya \\ University of Central Florida \\ Patrick LiKamWa \\ University of Central Florida
}

Find similar works at: https://stars.library.ucf.edu/facultybib2010 University of Central Florida Libraries http://library.ucf.edu

This Article is brought to you for free and open access by the Faculty Bibliography at STARS. It has been accepted for inclusion in Faculty Bibliography 2010 s by an authorized administrator of STARS. For more information, please contact STARS@ucf.edu.

\section{Recommended Citation}

Zakariya, Abdullah and LiKamWa, Patrick, "Monolithic quantum-well-tunable laser based on optical beam steering and area-selective intermixing" (2013). Faculty Bibliography 2010s. 4920.

https://stars.library.ucf.edu/facultybib2010/4920

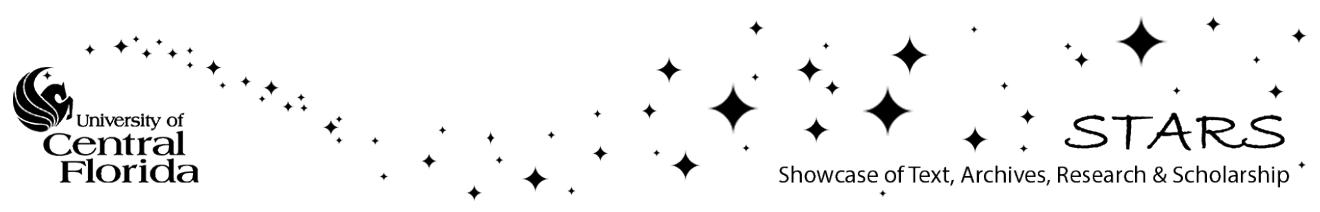




\title{
Optical Engineering
}

\section{Monolithic quantum-well-tunable laser based on optical beam steering and area- selective intermixing}

\author{
Abdullah Zakariya \\ Patrick LiKamWa
}




\section{Monolithic quantum-well-tunable laser based on optical beam steering and area-selective intermixing}

\author{
Abdullah Zakariya \\ Patrick LiKamWa \\ University of Central Florida \\ CREOL, The College of Optics and Photonics \\ Department of Electrical Engineering and \\ Computer Science \\ 4000 Central Florida Boulevard \\ Orlando, Florida 32816 \\ E-mail: azakariya@knights.ucf.edu
}

\begin{abstract}
A monolithic tunable laser on a quantum well $(\mathrm{QW})$ structure is demonstrated by integrating an optical beam-steering section with an area-selectively intermixed $\mathrm{QW}$ gain section. Wavelength tuning is achieved by guiding an amplified optical beam over an optical gain medium that consists of three laterally adjacent regions containing a quantum well that has been selectively intermixed to varying extents. The laser light output can be tuned over a total of a 17-nm wavelength range by separately injecting electrical current pulses to each of the two parallel contacts in the steering region and to the optical amplifier contact. @ 2013 Society of Photo-Optical Instrumentation Engineers (SPIE) [DOI: 10.1117/1.OE.52.9.096105]
\end{abstract}

Subject terms: quantum well lasers; semiconductor lasers diodes; tunable lasers; quantum wells intermixing; current-induced guiding.

Paper 130705 received May 13, 2013; revised manuscript received Aug. 13, 2013; accepted for publication Aug. 14, 2013; published online Sep. 9, 2013.

\section{Introduction}

Solid-state tunable laser diodes $\mathbb{\mathbb { Q }} \mathbf{Q}_{\text {are vital for environmental }}$ sensing applications such as atmospheric spectroscopy $\mathrm{EQ}_{\text {and }}$ chemical detection ${ }^{\mathbb{0}}$; moreover, they are also considered as an important factor in medical diagnosis ${ }^{\square}$ Tunable laser sources can be a central tool in any optical test that requires spectral analysis. In addition, tunable laser diodes present an attractive solution to many challenges in optical communication networks that require complex multiplexing schemes. Compact size and ease of fabrication using conventional semiconductor technology are also key to the realization of optoelectronic-integrated circuits with multiple functionalities. The previously reported scheme ${ }^{\mathbb{E}}$ used a vacancy-induced disordering of the quantum well $(\mathrm{QW})$ in the sampled grating region of a distributed Bragg reflector (DBR) laser diode to achieve a very wide range of tuning. However, it should be noted that the principle of the sample grating-DBR laser only allows continuous tuning over a small range of wavelengths, and the wide range of wavelength tuning takes place in multiple stages. In this article, we have fabricated and tested a tunable laser diode that combines the use of selective-area vacancy-induced intermixing and current-induced waveguiding. [1] Selective area intermixing of semiconductor quantum wells via dielectric capping and rapid thermal annealing produces an amplifier section with a broad range of optical gain spectrum, which is position-dependent. The amplifier section is monolithically integrated with an optical beam-steering section that guides the optical beam over the different parts of the gain section, which results in a laser chip with wavelength-tuning capabilities.

\section{Device Design and Theory}

\subsection{Device Design}

The wafer's active region consists of a single 4-nm thick GaAs quantum well sandwiched between two 5-nm thick

0091-3286/2013/\$25.00 () 2013 SPIE
$\mathrm{Al}_{0.25} \mathrm{Ga}_{0.75} \mathrm{As}$ barrier layers. These layers are surrounded by AlGaAs layers, each 150 -nm thick, where the concentration of aluminum is gradually increased from $\mathrm{Al}_{0.25} \mathrm{Ga}_{0.75} \mathrm{As}$ to $\mathrm{Al}_{0.55} \mathrm{Ga}_{0.45} \mathrm{As}$. These layers are in turn sandwiched by $\mathrm{Al}_{0.55} \mathrm{Ga}_{0.45} \mathrm{As}$ upper and lower cladding layers that are each 1.2- $\mu \mathrm{m}$ thick. The tunable laser device is designed on the monolithic structure consisting of two sections, a beam-steering section and an optical amplifier gain section, as shown in Fig. 11. The $20-\mu \mathrm{m}$ wide beam-steering section is bounded by two $10-\mu \mathrm{m}$ wide parallel contact stripes. Controlled levels of current injection into the parallel stripes of the steering section can confine and laterally steer the optical beam to remain in a specific location in the optical amplifier section. The optical amplifier gain section consists of three adjoining regions, each approximately $6-\mu \mathrm{m}$ wide to a total width of $20 \mu \mathrm{m}$.

The quantum well of these three regions of the amplifier gain section has been intermixed by varying amounts. The rest of the device $\mathrm{QW}$ is intermixed to the maximum extent corresponding to a blue shift of the electroluminescence peak wavelength of $32 \mathrm{~nm}$ in this particular case.

\subsection{Quantum Well Disordering/Intermixing}

Selective area QW disordering/intermixing is a low cost and a reliable postgrowth means of altering the bandgap energies of localized areas. This allows for monolithic device architectures containing multiple regions with different optical bandgaps within the same structure. Bandgap energies of the selectively intermixed regions are blue shifted by amounts that depend on the thickness of a dielectric capping film. $\mathrm{SiO}_{2}$ is one of the most common dielectric caps used for this process. The $\mathrm{SiO}_{2}$ film promotes the out diffusion of $\mathrm{Ga}$ atoms into that cap, which results in vacancies that migrate very quickly through the $\mathrm{QW}$ structure during rapid thermal annealing, and thereby, the vacancies are interchangeably filled by $\mathrm{Ga}$ and $\mathrm{Al}$ atoms, inducing intermixing of these constituent atoms from the well and the barrier materials. To determine the degree of bandgap change that occurs in the quantum well of our device, samples with different 


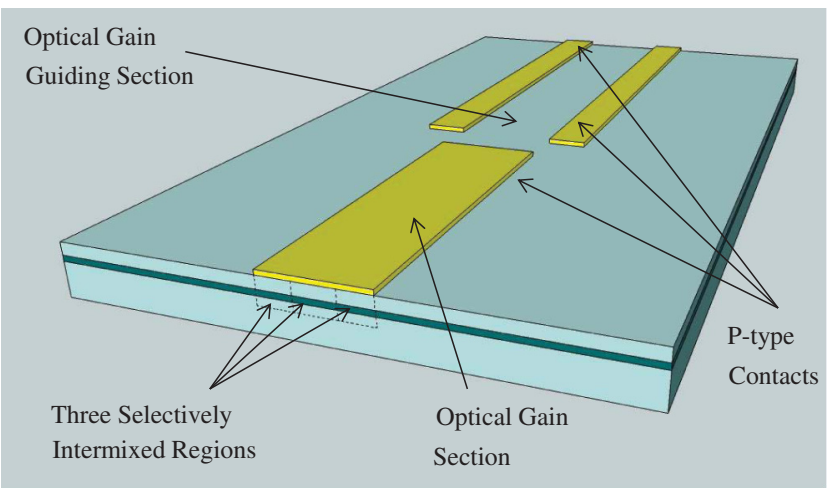

Fig. 1 Tunable laser design.

thicknesses of $\mathrm{SiO}_{2}$ capping films have been annealed at $975^{\circ} \mathrm{C}$ for $20 \mathrm{~s}$. The samples are then characterized using electroluminescence measurements. The results shown in Fig. Z indicate that the peak of the luminescence spectrum, which is $805 \mathrm{~nm}$ for the as-grown sample, is shifted to $800 \mathrm{~nm}$ for the uncapped sample, to $790 \mathrm{~nm}$ for the sample with $100-\mathrm{nm}$ thick $\mathrm{SiO}_{2}$, to $779 \mathrm{~nm}$ for the sample with a 200-nm thick $\mathrm{SiO}_{2}$, and to $772 \mathrm{~nm}$ for the sample capped with a $400-\mathrm{nm}$ thick $\mathrm{SiO}_{2}$ film.

To achieve the multiwavelength monolithic structure, separate regions of the device are disordered by different amounts. The optical amplifier gain section is divided into three adjacent regions, in which the quantum well has been selectively intermixed to varying extents using an uncapped region, a $100-\mathrm{nm}$ thick $\mathrm{SiO}_{2}$ capped region, and a $200-\mathrm{nm}$ thick $\mathrm{SiO}_{2}$ capped region. The remainder of the device is intermixed to the fullest extent by annealing with a 400nm thick $\mathrm{SiO}_{2}$ cap layer to minimize absorption of the laser beam in the steering section.

\subsection{Current-Induced Guiding}

Current-induced guiding of optical beams can be realized by independently forward-biasing the two parallel contact stripes of the beam-steering section. When electrical current is injected into each of the parallel contact stripes, a large density of electrons is accumulated in each of the quantum well region that lies directly underneath each of the metal contact stripes. As the electron concentration increases in the quantum well, they diffuse sideways and eventually almost fill out the QW region bounded by the two stripes. However, the lateral spread of the electrons can be controlled by carefully managing the magnitude of the electrical currents. The regions of the QW containing the largest concentration of electrons experience the largest decrease in refractive index through the band-filling (Burstein-Moss) effect. The channel between the two low refractive index regions becomes an optical channel waveguide. Therefore, by carefully adjusting the magnitudes of the injected currents, we can position the channel arbitrarily between the two contact electrodes, and thereby can guide an optical beam. ${ }^{13}$ We expect that as we guide the amplified optical gain into the intermixed gain regions, the tunable laser will operate at one of the three wavelengths of 800,790 , and $779 \mathrm{~nm}$, corresponding to the peak of the gain spectrum of that particular intermixed region of the optical amplifier gain section. Furthermore, by guiding the lasing beam at the interface between the selectively intermixed gain regions, the lasing wavelength is fully adjustable between the limits.

\section{Device Fabrication}

The masks used in this article were fabricated in-house using a NEOS laser photoplotter. A set of four masks was prepared to fabricate the final device. The fabrication process starts with the exposure of a negative photoresist coating, followed by the deposition of chromium alignment marks on the sample. These serve as fiduciary markers for the subsequent photolithographic steps. A $400-\mathrm{nm}$ thick layer of $\mathrm{SiO}_{2}$ is grown by plasma-enhanced chemical vapor deposition over the whole sample. The 400-nm cap remains over the whole device, except for the gain section which is subdivided into three regions. These three areas with different $\mathrm{SiO}_{2}$ thicknesses are defined via three consecutive steps of photolithography and reactive-ion etching. Each photolithographic step defines a $6-\mu \mathrm{m}$ wide window, followed by a reactive-ion etching step to etch down the $\mathrm{SiO}_{2}$ film to the required thickness, resulting in a gain section consisting of three distinct regions: (1) uncapped, (2) with 100-nm thick $\mathrm{SiO}_{2}$ cap, and (3) with 200-nm thick $\mathrm{SiO}_{2}$ cap. The sample is then annealed at $975^{\circ} \mathrm{C}$ for $20 \mathrm{~s}$ to achieve the three regions with different optical gain spectra, whereas the rest of sample that is capped with $400-\mathrm{nm}$ thick $\mathrm{SiO}_{2}$ film is intermixed to the fullest extent. The $\mathrm{SiO}_{2}$ film is then completely etched off using

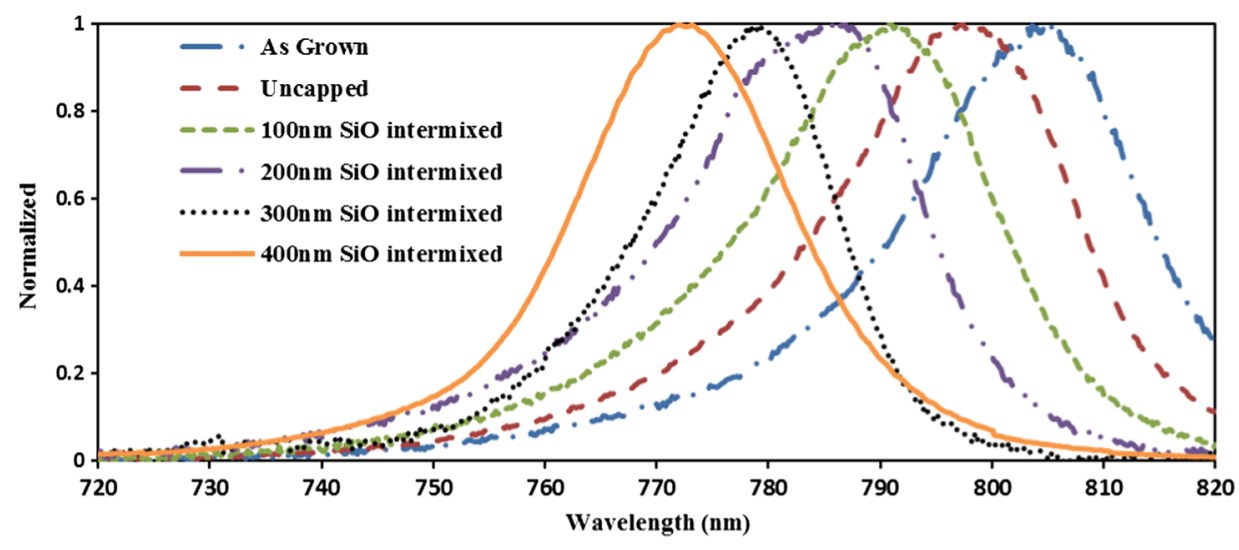

Fig. 2 Electroluminescence spectra of the QW structure for different $\mathrm{SiO}_{2}$ capping thicknesses. 

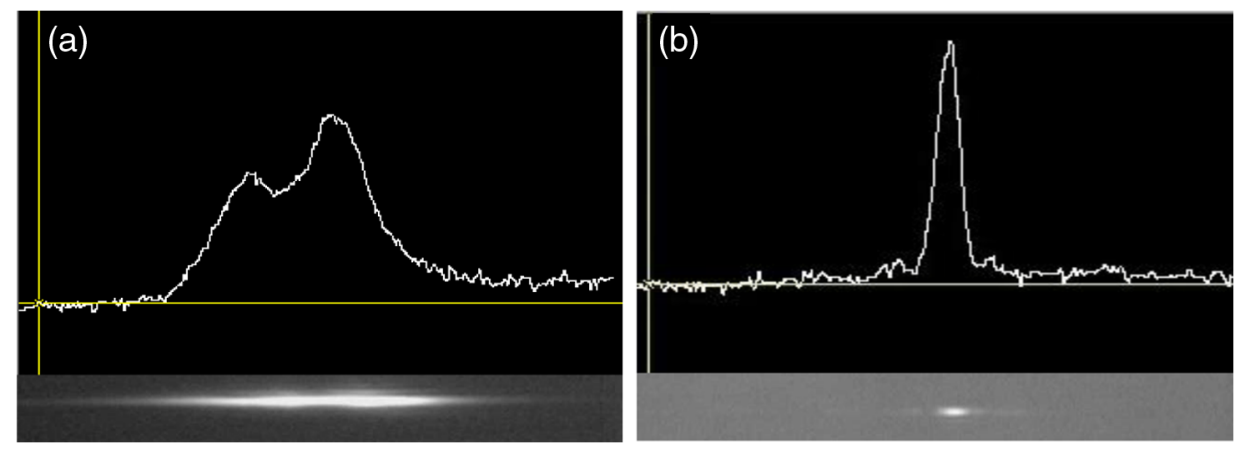

Fig. 3 (a) Output beam profile from the gain section for electrical injection that is below and (b) above thresholds.

buffered oxide etch. Finally, the top p-type contacts are delineated as two 10- $\mu \mathrm{m}$ wide parallel stripes for the steering section and a broad $20-\mu \mathrm{m}$ wide stripe over the gain section using photolithography, vacuum evaporation, and "lift-off," followed by annealing at $400^{\circ} \mathrm{C}$ for $1 \mathrm{~min}$. The alignment of the two parallel metal contacts is such that the stripes are directly on the top of the most heavily intermixed regions of the QW structure to perform optical beam guiding and steering. The broad contact allows current to be injected into the optical amplifier section comprising the three QW regions that have been intermixed to lesser extents. The sample is then thinned down by lapping and polishing the backside of the substrate, and finally, the n-type contact metal is evaporated on the substrate and annealed. The photolithography mask is designed such that we can cleave the device's gain section to any desired length in the range of $100 \mu \mathrm{m}$ to $1 \mathrm{~mm}$.

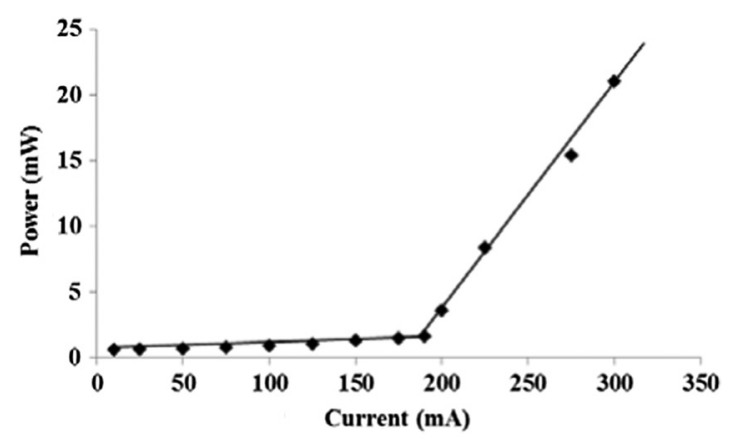

Fig. 4 Gain section L-I curve.

\section{Testing and Results}

The device was cleaved to a total length of $750 \mu \mathrm{m}$ with a $250-\mu \mathrm{m}$ long gain section and mounted on a copper plate. A setup consisting of three independently controlled probes was used to characterize the device. One probe injects current into the gain section, and the other two are used to connect to the steering contact stripes. The device characterization begins with the investigation of the gain section by itself. First, a pulsed current of $180 \mathrm{~mA}$ is injected into the gain section, and the output facet electroluminescence is imaged onto a charge-coupled device camera by a $40 \times$ microscope objective lens and simultaneously collected and refocused into an Ocean Optics, Orlando, FL, USB4000 spectrometer. From the output beam profile of the electroluminescence shown in Fig. B(a), it is apparent that a larger accumulation of electrons occurs in the least intermixed region as evidenced by a stronger emission there. This is to be expected, since electrons will naturally occupy the lowest energy levels in the least intermixed region of the quantum well. The current is then increased slightly above the threshold current of $190 \mathrm{~mA}$ to make the device lase. Once the device starts to lase, the output beam is observed to narrow down significantly in Fig. 3(b), and its lateral position is found to correspond to that of the highest intensity of the electroluminescence beam. Furthermore, the laser wavelength is at $800 \mathrm{~nm}$, which corresponds to the peak of the gain in the least disordered region of the amplifier section.

The device exhibited a threshold current of $190 \mathrm{~mA}$ with a calculated threshold current density $J$ 'th of $3.8 \mathrm{kA} / \mathrm{cm}^{2}$ and a total loss coefficient $\alpha$ of $4.81 \mathrm{~dB} / \mathrm{cm}$. Figure $\square$ shows the L-I [optical output (L) as a function of the current
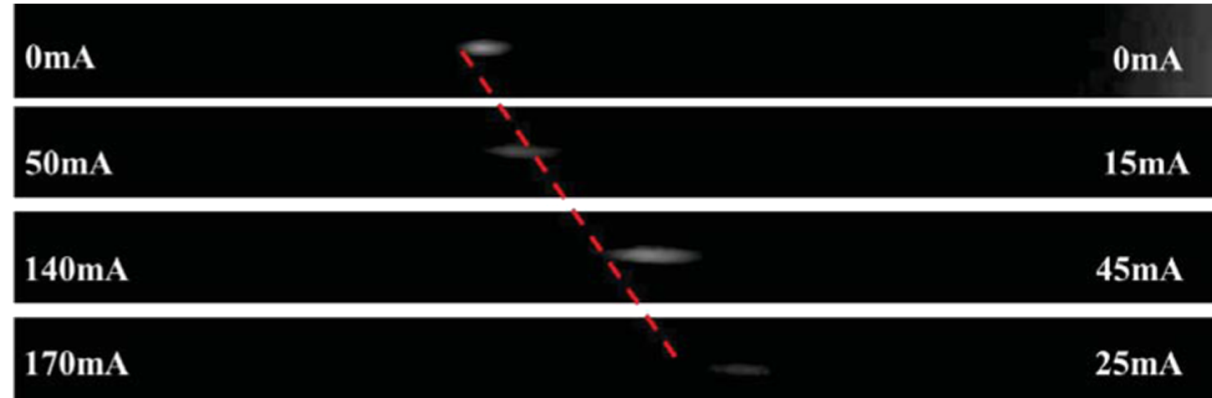

Fig. 5 Laser beam-steering sequence as viewed from the gain section facet, while different current values are injected into the steering section. 
Table 1 Wavelength tunability of current injection values.

\begin{tabular}{lccc}
\hline \hline $\begin{array}{c}\text { Gain } \\
(\mathrm{mA})\end{array}$ & $\begin{array}{c}\text { Left contact } \\
(\mathrm{mA})\end{array}$ & $\begin{array}{c}\text { Right contact } \\
(\mathrm{mA})\end{array}$ & $\begin{array}{c}\text { Wavelength } \\
(\mathrm{nm})\end{array}$ \\
\hline 220 & 0 & 0 & 800 \\
210 & 12 & 0 & 799 \\
200 & 50 & 15 & 798 \\
200 & 70 & 25 & 795 \\
200 & 83 & 31 & 794 \\
195 & 104 & 30 & 793 \\
190 & 110 & 35 & 792 \\
183 & 120 & 43 & 791 \\
170 & 140 & 50 & 787 \\
166 & 150 & 40 & 786 \\
153 & 150 & 45 & 785 \\
149 & 165 & 25 & 784 \\
143 & 170 & & \\
\hline \hline
\end{tabular}

(I)] curve of the gain section. In order to change the lasing wavelength, the optical beam needs to be pushed away by steering the optical beam from the least intermixed region into the region that has been intermixed to a larger extent.

Pulsed current injected into the gain section is first increased to above threshold condition to cause the device to lase. Electrical pulsed currents are then injected into the parallel contacts of the beam-steering section with an increasingly higher value in the contact that is adjacent to the least intermixed gain region. As the current value is slowly increased, the lasing beam is gradually forced toward the more heavily intermixed region, as shown in Fig. 5.

In our experiment, a total wavelength tuning of $17 \mathrm{~nm}$ is achieved, corresponding to the pulsed injected current values in Table 1, as the laser beam is steered between the least intermixed to the most heavily intermixed section, as shown in Fig. 6 .

\section{Discussion and Conclusion}

To achieve wavelength tunability and output power stability for the tested device, each stripe was injected with current that was adjusted independently so as to obtain the desired laser output wavelength, while maintaining almost constant output power, as shown in Fig. 6. When the device was first designed, it was initially anticipated that the wavelength-tuning range would be determined by the gain spectra from the three intermixed regions with electroluminescence peaks of 800,790 , and $779 \mathrm{~nm}$, respectively. However, the fabricated device produced a wavelength tuning of $17 \mathrm{~nm}$ ranging from 800 to $783 \mathrm{~nm}$, instead of the expected $779 \mathrm{~nm}$. This is most likely due to the lower gain coefficient of the more intermixed QW. Table 1 shows a set of injected current values that resulted in the reported wavelength tuning.

Laser operation at wavelengths between the gain peaks of the three regions is attainable because at the interface between the intermixed regions in the gain section, there is a gradient in the degree of QW intermixing. The precise positioning of the laser beam at the interface between the intermixed regions allows for a fine wavelength tuning. Although the speed of the wavelength switching has not been measured experimentally, it is expected to be only limited by carrier recombination lifetime, and therefore, should respond on a nanosecond time scale. From the emission spectra shown in Fig. 6, it is clear that the current injected through the stripes that control the beam-steering affect the overall optical gain of the structure. Therefore, in order to maintain nearly constant emitted laser power, the current in the main gain section has to be changed accordingly. Although the output beam profile that is obtained is shown in Fig. B(b), the beam quality of the laser beam from this device has not been studied because the main focus of this report is wavelength tunability. However, once wavelength tunabilty has been perfected, the output beam quality from the device will be evaluated, since it is an important factor in optical telecommunication systems.

In conclusion, we have fabricated and experimentally investigated a tunable laser based on combined beam steering in a passive region and optical gain guiding over three selectively intermixed gain regions. The device output had a total tuning range of 17-nm wavelength, which was achieved by controlling the current values that were injected into the three contact stripes. For future works, to make the device applicable to commercial use, a single-mode waveguide will be fabricated on the side of the beam-steering section that is opposite to the gain section. The QW in the single mode waveguide will be highly intermixed, so as to present minimal absorption to the emitted laser beam. In this manner,

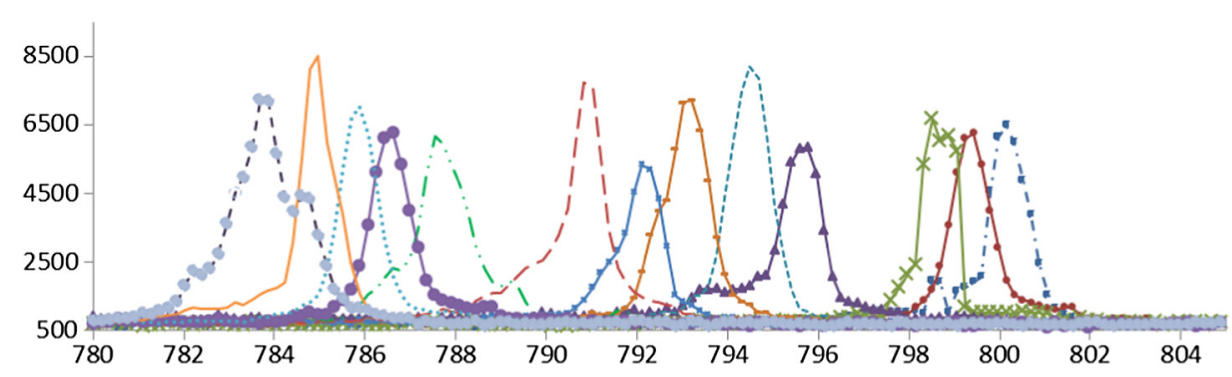

Fig. 6 Spectra of the laser emission corresponding to different current conditions. 
the laser beam emitted from the single-mode laser will remain stationary and will have a $\mathrm{TEM}_{00}$-mode quality.

\section{References}

1. M. Aoki et al., "InGaAs/InGaAsP MQW electroabsorption modulator integrated with a DFB laser fabricated by band-gap energy control selective-area MOCVD," IEEE J. Quantum Electron. 29(6), 20882096 (1993).

2. H. Ishii et al., "Quasicontinuous wavelength tuning in super-structuregrating (SSG) DBR lasers," IEEE J. Quantum Electron. 32(3), 433-441 (1996).

3. V. Jayaraman, Z. M. Chuang, and L. A. Coldren, "Theory, design, and performance of extended tuning range semiconductor lasers with sampled gratings," IEEE J. Quantum. Electron. 29(6), 1824-1834 (1993).

4. J. Werner et al., "Integrated external cavity GaAs/AlGaAs lasers using selective quantum well disordering," Appl. Phys. Lett. 55(6), 540-541 (1989).

5. R. Phelan et al., "A novel two section tunable discrete mode Fabry-Pèrot laser exhibiting nanosecond wavelength switching," IEEE J. Quantum Electron. 44(4), 331-337 (2008).

6. E. J. Skogen et al., "Postgrowth control of the quantum-well band edge for the monolithic integration of widely tunable lasers and electroabsorption modulators," Sel. Top. Quantum Electron. 9(5), 1-8 (2003).

7. J. W. Raring et al., "Widely tunable negative-chirp SG-DBR laser/EAmodulated transmitter," J. Lightwave Technol. 23(1), 80-86 (2005).

8. M. N. Fiddler et al., "Laser spectroscopy for atmospheric and environmental sensing," Sensors 9(12), 10447-10512 (2009).

9. M. G. Alleny, "Diode laser absorption sensors for gas-dynamic and combustion flows," Meas. Sci. Technol. 9(4), 545-562 (1998).

10. R. S. Harmon et al., "Laser-induced breakdown spectroscopy - an emerging chemical sensor technology for real-time field-portable, geochemical, mineralogical, and environmental applications," Appl. Geochem. 21(5), 730-747 (2006).

11. S. Svanberg," "Environmental and medical applications of photonic interactions," Phusica Scripta T110, 39-50 (2004).

12. J. H. Marsh, "Quantum well intermixing," Semicond. Sci. Technol. 8(6), 1136-1155 (1993).
13. X. Dong et al., "Current-induced guiding and beam steering in active semiconductor planar waveguide," IEEE Photonics Technol. Lett. 11 (7), 809-811 (1999).

14. J. Manning, R. Olshansky, and C. B. Su, "The carrier-induced index change in AlGaAs and $1.3 \mu \mathrm{m}$ InGaAsP diode lasers," IEEE $J$. Quantum Electron. 19(10), 1525-1530 (1983).

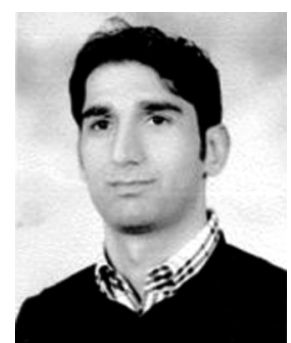

Abdullah Zakariya works for the Ministry of Interior Kuwait: Information Technology \& Telecommunication sector. He received his MS in electrical engineering at Florida International University, Miami, Florida in 2007 and BS in electrical engineering from Florida Institute of Technology, Melbourne, Florida. $\mathrm{He}$ is currently completing his $\mathrm{PhD}$ in electrical engineering at the University of Central Florida at CREOL and FPCE-The College of Optics and Photonics, Orlando, Florida. He is conducting research on semiconductor tunable lasers, multiwavelength LEDs, and QW disordering/intermixing.

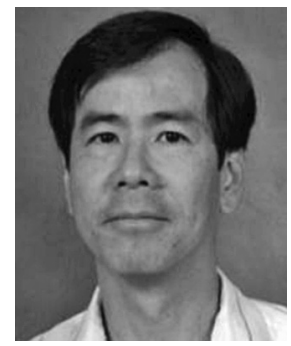

Patrick LiKamWa received his $\mathrm{PhD}$ in electrical engineering at the University of Sheffield, Sheffield, England and is an associate professor of optics and electrical and computer engineering at UCF, Orlando, Florida. He is currently pursuing research on the design, fabrication, and testing of novel all-optical switching devices using III$\mathrm{V}$ multi-quantum well semiconductors, and the integrating high-speed optical and optoelectronic devices to form monolithic integrated optical circuits for high-data throughput optical networks. 